\title{
El sistema bibliotecario de la Universidad Nacional Autónoma de México: ¿un consorcio?
}

\author{
Por Margarita Lugo Hubp y Raúl Novelo Peña
}

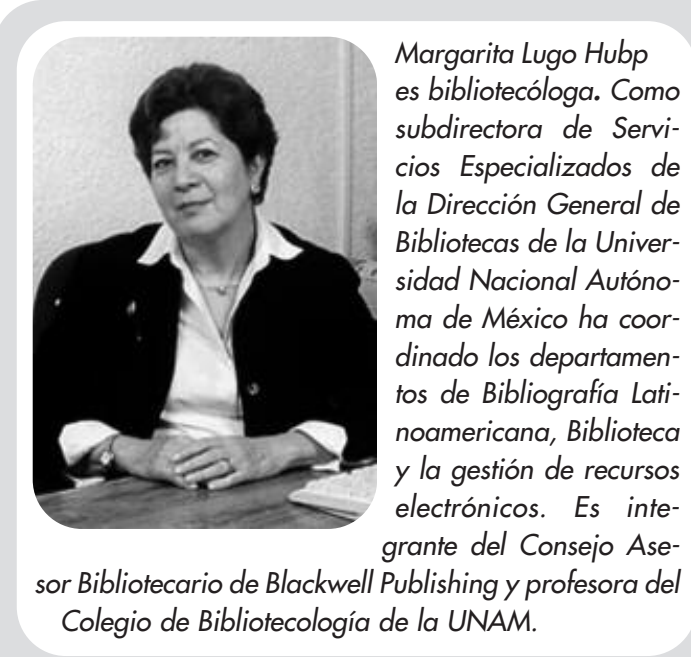

Resumen: La experiencia acumulada por la UNAM en la adquisición de documentos impresos y bases de datos en discos compactos le permitió establecer acciones para llevar a cabo la adquisición centralizada y compartida de los recursos electrónicos, teniendo como objetivos la racionalización del presupuesto y evitar la duplicidad que se tuvo en la compra de revistas impresas y bases de datos en cd-rom. En este trabajo exponemos cómo el sistema bibliotecario de la UNAM adquiere recursos electrónicos de forma similar a algunos consorcios de bibliotecas, sin llegar a ser un consorcio en sí mismo.

Palabras clave: Adquisición compartida, Consorcios, Recursos electrónicos, UNAM.

Title: The library system of the Universidad Nacional Autónoma de México: a consortium?

Abstract: UNAM's acquired experience with the acquisition of print documents and cd-rom databases has allowed it to establish actions for carrying out centralised and shared electronic resource acquisition. Its goals are to rationalise spending and to avoid the duplicate subscriptions that occurred with print journals and cd-rom databases. In this article we explain how the UNAM's library system acquires electronic resources in ways similar to some $l i$ brary consortia, even though it does not constitute a consortium itself.

Keywords: Shared acquisition, Consortia, Electronic resources, UNAM.

Trabajo presentado en el congreso Amigos 2004: consorcios en la sociedad del conocimiento, organizado por la Red Nacional de Cooperación Bibliotecaria y celebrado del 12 al I4 de febrero de 2004 en la Universidad de las Américas, Puebla, México. 
LA UNIVERSIDAD NACIONAL AUTÓNOMA DE LA MÉXICO (UNAM) es una de las instituciones de educación superior más grandes e importantes de América Latina. Para el año académico 2003-2004 la matrícula de alumnos inscritos fue de 269.143, de los cuales 104.554 pertenecen al bachillerato, 145.602 al nivel técnico, propedéutico y de licenciatura y 18.987 al postgrado. El personal académico (ayudantes de profesor, profesores de asignatura y de carrera, investigadores y técnicos académicos) que trabajó en la $U N A M$ en el 2004 ascendió a 32.498 de los cuales 11.236 trabajaron a tiempo completo en la institución'.

Para apoyar las actividades del personal académico y la formación de sus estudiantes, la universidad cuenta con un sistema bibliotecario integrado por 138 bibliotecas de las cuales 49 corresponden a centros e institutos de investigación, 7 a unidades multidisciplinarias, 44 a escuelas y facultades, 16 al bachillerato y 22 al servicio de extensión universitaria y administración ${ }^{2}$. En cuanto a la distribución geográfica de las mismas, 116 se ubican en la zona metropolitana de la ciudad de México y las otras 22 en 10 estados de la República Mexicana; estas últimas, en la mayoría de los casos son unidades pequeñas y especializadas que apoyan actividades de investigación y de formación en los postgrados.

El acervo que albergan estas bibliotecas es de $5.532 .697^{3}$ volúmenes de obras monográficas, 59.662 títulos de revistas y 690.256 ejemplares de tesis ${ }^{4}$.

Algunas bibliotecas, además, resguardan importantes colecciones especiales integradas por libros raros y valiosos, así como una cantidad considerable de otros tipos de materiales como vídeos, fotografías, discos compactos, folletos, ma-

pas, microformatos, etc. El control y difusión de la mayor parte de estos materiales se realiza con el apoyo de los catálogos Librunam, Seriunam y Tesiunam.

La riqueza de sus colecciones y el acceso que se ofrece a ellas hace del sistema bibliotecario de la UNAM un apoyo importante no sólo a las actividades académicas de la universidad, sino también a la de numerosos lectores que acuden y solicitan constantemente dichas colecciones. De acuerdo a los datos disponibles, en el año 2001, tanto los usuarios internos como los externos asistieron a las bibliotecas en 21.332.340 ocasiones; consultaron en las salas de lectura 26.355.214 volúmenes y solicitaron en préstamo a domicilio 13.415.723 volúmenes 5 .

\section{«La UNAM cuenta con un sistema bibliotecario integrado por 138 bibliotecas que albergan un acervo de 5.532.697 volúmenes de obras monográficas, $\mathbf{5 9 . 6 6 2}$ títulos de revistas y 690.256 volúmenes de tesis»}

Si bien la UNAM ya tenía una vasta experiencia en la adquisición, control bibliográfico y circulación de documentos impresos, ahora los nuevos soportes de información trajeron consigo un reto más que enfrentar para incorporarlos al quehacer cotidiano de las actividades sustantivas de la universidad.

Hubo diversos aspectos que incidieron en la toma de decisión para establecer acciones que llevaran a la adquisición centralizada y compartida de recursos electrónicos, pero entre los más importantes estaban la necesidad de racionali- zar los recursos y evitar la duplicidad que se había observado en la compra de revistas impresas y bases de datos en disco compacto. Tratemos de imaginar el esfuerzo de 138 responsables de bibliotecas intentando negociar individualmente o en pequeños grupos la compra de recursos electrónicos, varios de ellos seguramente duplicados; también visualicemos el trabajo que implicaría en lo individual administrar la conexión a estos recursos. Por ello, a partir de 1997 la Dirección General de Bibliotecas (DGB) ha asumido el liderazgo en la negociación para adquirir y administrar estos recursos electrónicos.

\section{Selección}

Cuando se inicia la compra centralizada de revistas electrónicas se establece como criterio de selección adquirir aquellos títulos cuya versión impresa se tenía en las bibliotecas departamentales, principalmente en las de los centros e institutos de investigación. Esto garantizaba que un grupo de académicos ya había evaluado y determinado su pertinencia. También hubo otros títulos de los cuales no se tenía la edición en papel pero que por sus contenidos y características podrían ser de utilidad para algún grupo de universitarios; en este caso también fueron seleccionados y se estableció que se observaría el uso para determinar si era conveniente continuar o no con el acceso o suscripción. Este esfuerzo institucional se inició con 600 títulos de revistas y actualmente se tienen cerca de 7.300.

En el caso de las bases de datos se da una situación similar al de las revistas, la $D G B$ también seleccionó de manera inicial aquellos títulos cuyas versiones ya se habían adquirido en disco compacto. Nuevamente esta decisión tuvo el sustento de que una comunidad de usuarios especializados ya había 
realizado la evaluación correspondiente para determinar la pertinencia de dichas bases de datos. En una primera etapa se adquirieron $30 \mathrm{y}$ actualmente se cuenta con 162 títulos.

Como anotamos con anterioridad, en esta primera fase la selección de títulos de revistas y bases de datos en línea la realizó la $D G B$ teniendo en cuenta los que ya existían en formato impreso o en cdrom. Hoy son las bibliotecas departamentales a través de sus comisiones de bibliotecas autónomas quienes determinan la conveniencia y/o necesidad de continuar con las suscripciones a estos recursos, de sustituir o de incorporar otros que apoyen las actividades sustantivas de nuestra universidad. En este punto, la función principal de la $D G B$ ha sido vigilar que cualquier decisión de las bibliotecas departamentales, por ejemplo en las cancelaciones, no afecte a otras, evitar la duplicación de títulos y asegurar que se aprovechen al máximo los presupuestos destinados a la información digital.

Por otra parte, ha sido necesario crear otras instancias que tengan entre su funciones evaluar y recomendar la adquisición de otros recursos que por sus características y cobertura amplia así lo ameritan, como son: obras de consulta generales, bases de datos multidisciplinarias, sistemas agregadores, paquetes de revistas de una determinada editorial, paquetes de libros electrónicos comercializados por una compañía, etc. Entre ellas podemos mencionar el Comité evaluador de revistas y bases de datos electrónicas (Cerbde) integrado por diversos especialistas en evaluación, bibliotecología, ciencias de la información e informática y que funciona como órgano asesor de la $D G B$. Este comité se instaló el 2 de abril de 2002 con la finalidad de conocer, evaluar y recomendar la renovación, cancelación y nueva

\begin{tabular}{|c|c|c|c|}
\hline Año & Costo de bases de datos (US\$) & N. dep. beneficiadas & Plataforma \\
\hline $1994^{6}$ & 948.534 US\$ & 45 & cd-rom \\
\hline $1996^{6}$ & 907.176 US\$ & 47 & cd-rom \\
\hline $1999^{7}$ & 1.369 .888 US\$ & $\begin{array}{c}139 \text { y toda la } \\
\text { comunidad }\end{array}$ & En línea \\
\hline
\end{tabular}

Cuadro 1: costo de las suscripciones a bases de datos y dependencias beneficiadas (suscripciones en la UNAM) suscripción de recursos de información digital (revistas y bases de datos); todo ello con la intención de optimizar el gasto asignado para la compra de estos materiales y propiciar su consulta por parte de la comunidad universitaria.

\section{«En una primera etapa la selección de títulos de revistas y bases de datos en línea la realizó la DGB basándose en los que ya existían en formato impreso o en cd-rom; hoy lo hacen las bibliotecas departamentales a través de sus comisiones de biblioteca autónomas»}

Algunas de las principales funciones previstas para el Cerbde son: realizar estudios de costo-beneficio de los servicios y recursos que se suscriben; establecer parámetros e indicadores para la evaluación de la información digital; diseñar una metodología para realizar estudios y propuestas para las cancelaciones de revistas impresas con más de una suscripción en la
UNAM. Los integrantes del comité forman, además, parte de alguno de sus tres grupos operativos de trabajo: Evaluación de recursos comerciales; Evaluación de recursos alternativos y Racionalización de recursos asignados a revistas.

\section{Adquisición}

Las primeras adquisiciones online las realizó el Centro de Información Científica y Humanística antes de incorporarse como parte de la $D G B$ en febrero de 1997. Estos títulos fueron: Elsevier Science, Cambridge Scientific Abstracts Complete Science Collection (28 bases de datos) y Swetscan. El pago de las suscripciones se realizó con la partida presupuestal para revistas científicas y técnicas, la cual zada en el Departamento de Suscripciones de esta dirección. Posteriormente, la $D G B$ realizó una sustitución de las bases de datos que se encontraban en formato cd-rom por la edición online utilizando los recursos que ya se tenían invertidos en este tipo de material.

En el cuadro 1 se muestran datos del costo de las bases de datos en cd-rom y en línea, así como el número de dependencias benefiha estado casi totalmente centrali-

\begin{tabular}{|c|cc|c|c|c|}
\hline Año & Número de títulos & US\$ & T.C. ${ }^{*}$ & Moneda nacional \\
\hline 1999 & 2800 & 66 & 1.369 .888 US $\$$ & 10.23 & $\$ 14.014 .292$ \\
\hline 2000 & 6000 & 106 & 2.231 .043 US $\$$ & 9.58 & $\$ 21.382 .840$ \\
\hline 2001 & 6400 & 140 & 2.720 .652 US $\$$ & 9.51 & $\$ 25.885 .535$ \\
\hline 2002 & 6500 & 142 & 2.806 .754 US $\$$ & 9.38 & $\$ 26.321 .699$ \\
\hline 2003 & 7300 & 160 & 2.580 .571 US $\$$ & 10.52 & $\$ 27.150 .838$ \\
\hline
\end{tabular}

* Tipo de cambio que se utilizó para hacer la conversión de dólares americanos, a pesos mexicanos.

Cuadro 2: crecimiento e inversión realizados a revistas y bases de datos electrónicas en la UNAM.

Fuente: Dirección General de Bibliotecas. Subdirección de Servicios Especializados/Departamento de Suscripciones. UNAM, 2003. 
ciadas (escuelas, institutos) con estos recursos.

En cuanto al pago de estos recursos, poco a poco la responsabilidad se ha ido transfiriendo a las bibliotecas departamentales que se han beneficiado de manera directa con estos accesos y que han asumido los costos individualmente o en pequeños grupos. En los casos de la adquisición compartida, y cuando el proveedor requiere un solo pago, ha sido necesario establecer procedimientos y mecanismos que permitan transferir y concentrar los recursos económicos para posteriormente hacer el pago en una exhibición.

En el cuadro 2 se muestra el crecimiento del gasto que se ha realizado para la adquisición de revistas y bases de datos, así como las diferencias en el tipo de cambio.

\section{Negociación con proveedores}

Las primeras negociaciones que tuvo que emprender la $D G B$ con los proveedores de estos recursos fueron bastante arduas y complejas por lo que implicaba gestionar accesos para una cantidad considerable de bibliotecas y a costos que estuvieran acordes a su uso real. Por un lado los proveedores proponían esquemas en los cuales el precio se fijaba considerando el número de estudiantes o de personal académico o de computadoras; por otro, la $D G B$ consideraba que un esquema equitativo eran las licencias de acceso validado por dirección Ip para toda la UNAM y con un número determinado de usuarios simultáneos. A la fecha, ésta es la fórmula que ha demostrado ser la más adecuada, ya que no requiere un gasto excesivo y los usuarios que utilizan cada uno de los recursos, generalmente, tienen el acceso cuando lo necesitan.

A manera de ejemplo, para poder calcular el número de licencias conveniente para cada base de datos, se revisaron los datos de la matrícula de la UNAM, por área del conocimiento y nivel educativo, así como las cifras sobre número de académicos de tiempo completo y por asignatura en la institución. Además, se aceptó la fórmula sugerida por algunos especialistas: una licencia por cada 2.000 usuarios potenciales, aproximadamente.

\section{«En los casos de la adquisición compartida y cuando el proveedor requiere un solo pago, ha sido necesario establecer procedimientos que permitan transferir los recursos económicos para posteriormente hacer el pago en una exhibición»}

Los convenios que se establecieron en esta etapa fueron muy importantes porque permitieron sentar las bases para las futuras negociaciones que llevarían a cabo también las bibliotecas departamentales y, además, se dio la oportunidad de dar conocer a los proveedores las características reales de la $U N A M$, evitando así propuestas que sólo tomaba en cuenta la magnitud de nuestra universidad. Entre las primeras negociaciones más significativas para la UNAM se puede mencionar la que se inició con Elsevier, cuya problemática es la inestabilidad del número de títulos ya que se presentan fusiones, cambios de título e ISSN, de periodicidad y títulos que cesan, lo cual dificulta el establecimiento claro de la colección y el cumplimiento de la empresa en dar acceso durante el año entero a todo lo que se paga. A esto se debe añadir el movimiento de suscripciones que realizan año tras año las bibliotecas.
Otro de los casos destacados fue la suscripción del sistema Ovid Full Text debido a que constituyó la primera adquisición compartida. Las Facultades de Estudios Superiores Cuautitlán e Iztacala aportaron más del $35 \%$ del costo desde el inicio de la suscripción.

Actualmente, en los convenios que han establecido algunas de las bibliotecas departamentales, la $D G B$ ha estado presente en las negociaciones de manera directa o indirecta para garantizar que las propuestas de los proveedores sean las más adecuadas para la UNAM y por tanto los accesos contratados estén disponibles para todos los usuarios que así lo requieran.

\section{Acceso}

La facilidad de acceso que encierran los formatos electrónicos es, sin duda, una de sus principales ventajas. La UNAM, al ir adquiriendo sus recursos fue construyendo un gran acervo electrónico que propició la creación del proyecto de Biblioteca Digital de la $D G B$. Los recursos están organizados en una página web en la que de manera sistemática se van incluyendo los accesos a toda la información suscrita. Esta biblioteca, además de ofrecer la consulta sin costo para el usuario final, es un medio para la obtención de documentos en texto completo para todos los universitarios y “(...) pretende establecer políticas, procedimientos y flujos de trabajo basados en normas y estándares internacionales que conformen estructuras sólidas sobre el comportamiento de la información digital y sobre todo contribuir a la conformación de una Biblioteca Universitaria Digital"s. El reto es integrar cada vez de mejor manera una gran cantidad de información, para que las interfaces que el usuario utilice sean más sencillas y a la vez le reporten resultados relevantes y precisos.

http://dgb.unam.mx/ 


\section{El Comité de libro electrónico}

Si bien la UNAM desde hace cinco años ha estado trabajado en la edición electrónica y digital de documentos, la experiencia en la adquisición de libros electrónicos a través de proveedores es relativamente reciente. Se han tratado de aprovechar los conocimientos adquiridos con las revistas electrónicas y las bases de datos.

Para aunar y capitalizar este conocimiento y contar, además, con un órgano de consulta, la $D G B$ creó en febrero de 2002 el Comité de libro electrónico con los objetivos de generar y regular las políticas y los lineamientos para el desarrollo de una colección de este tipo de documento.

Para integrar el comité se invitó a un grupo de coordinadores de bibliotecas departamentales de los niveles de bachillerato, licenciatura y postgrado e investigación. Está integrado por los subcomités de selección, adquisición y negociación con los proveedores; infraestructura tecnológica; control bibliográfico; uso y evaluación del servicio; marco legal; y, financiamiento. Estos subcomités se formaron para impulsar al comité en el logro de su misión: "satisfacer las necesidades de información por medio de la selección, adquisición y arrendamiento de libros electrónicos, además de su organización, acceso y difusión en el sistema bibliotecario de la UNAM, a través de la innovación, calidad y servicio, a fin de consolidar la excelencia académica".

A lo largo de casi dos años ha realizado diversas actividades entre las que se encuentran las propuestas de políticas relacionadas con el libro electrónico; la firma de un convenio con el Fondo de Cultura Económica $(F C E)$ para tener acceso a las colecciones electrónicas de esta editorial; la compra o

arrendamiento de estos materiales para toda la universidad (NetLibrary, Safari, Marcel Dekker, Kluwer, etc.); y la creación de un catálogo que también sirve como vínculo para la consulta de estos libros (Libeunam).

\section{Los consorcios de bibliotecas y el sistema bibliotecario de la UNAM}

Estas agrupaciones son definidas como "asociación de empresas para defender intereses comunes" 10 constituidas por dos o más instituciones afines. En el ámbito bibliotecario los consorcios son creados con el fin de realizar tareas de cooperación de distintos tipos: compartir recursos, ampliar sus opciones de acceso a la información, optimizar sus presupuestos, o para realizar actividades más específicas que beneficien a todos los participantes.

\section{«El sistema \\ bibliotecario de la UNAM, conjuntamente con la DGB, participa \\ en actividades de selección y adquisición de recursos electrónicos semejantes a las que realizan algunos consorcios de bibliotecas, participando en actividades cooperativas y tratando siempre de optimizar los presupuestos asignados a dichos recursos»}

Si bien el sistema bibliotecario de la $U N A M$ en sentido estricto no conforma ningún consorcio para la selección y adquisición de recursos electrónicos, sí realiza actividades semejantes a las que llevan a cabo las instituciones que se han agrupado para desarrollar acciones cooperativas, tratando siempre de optimizar los presupuestos destinados a dichos recursos. Además de las tareas de selección y adquisición que ya se han mencionado en estas notas, consideramos importante señalar que la $D G B$ cuenta con datos precisos acerca de los usuarios a los que va dirigida la información, por nivel y área de conocimiento, y también se mantienen actualizados los datos relativos a los títulos de revistas que se suscriben con cada editor. En el caso de las bases de datos, además del control del uso y de las suscripciones, se realiza el monitoreo diario para asegurar su funcionamiento $y$ se preparan reportes de evaluación cuando se requieren.

\section{Algunas conclusiones}

En nuestros días, la cooperación bibliotecaria en el ámbito de la educación superior resulta indispensable para lograr el desarrollo de colecciones de acuerdo a las necesidades de los usuarios. Los aumentos en el costo anual de las revistas académicas y la falta de incremento en los presupuestos de las universidades propician la frecuente cancelación de suscripciones y con ello el deterioro de las colecciones de apoyo a la docencia y la investigación.

Desde hace varios años la $U N A M$ ha contado con los recursos económicos suficientes para la adquisición de materiales bibliográficos que apoyan sus actividades sustantivas y que por extensión benefician también a otras universidades e instituciones de investigación.

Los trabajos de racionalización y cooperación que se han dado entre el sistema bibliotecario y la $D G B$ en los últimos años han permitido que se mantengan estables las colecciones electrónicas básicas. Esta estabilidad ha facilitado que la $D G B$ integre bancos de da- 
tos de usuarios y recursos, incluyendo las modalidades en las cuales se tiene contratado el acceso, los costos, las fechas de cobertura $\mathrm{y}$ otros datos fundamentales para facilitar la implementación de consorcios, acuerdos o grupos de compra con otras instituciones de educación superior.

Tanto el Comité evaluador de revistas y bases de datos electrónicas como el de libro electrónico han consolidado el trabajo requerido para desarrollar adecuadamente dichos acervos, de ahí la importancia y trascendencia de su creación $\mathrm{y}$ funcionamiento.

En síntesis, el sistema bibliotecario de la UNAM no es un consorcio pero sí lleva a cabo diversas actividades, coordinadas por la $D G B$, que le han permitido beneficiar a las unidades de información que lo integran.

Para finalizar, es importante destacar que lo anteriormente expuesto ha permitido que la UNAM acumule información y una vasta experiencia que le ha servido como base para establecer convenios o acciones de cooperación con otras instituciones en la selección, adquisición y distribución de información electrónica.

\section{Notas}

1. Universidad Nacional Autónoma de México. Agenda estadística 2004. México: UNAM, 2004, pp. 19-20.
2. Ibidem. p. 255.

3. Ibidem, p. 260.

4. "El sistema bibliotecario de la UNAM en cifras: radiografía del sistema 2001". En: Biblioteca universitaria: revista de la Dirección General de Bibliotecas de la UNAM, 2002, enerojunio, v. 5, n. 1, pp. 61-65.

5. Universidad Nacional Autónoma de México. Dirección General de Bibliotecas. Censo estadístico 2001. México, 2002.

6. Hernández Mondragón, Alma Rosa, [et al.]. "Perspectivas en el manejo de información electrónica en la UNAM". En: Reunión de revistas académicas y de investigación, 1997, p. 101.

7. Universidad Nacional Autónoma de México. Dirección General de Bibliotecas. Departamento. de Suscripciones. Concentrado de gastos. México, 1999. [Documento interno de trabajo].

8. Universidad Nacional Autónoma de México. Dirección General de Bibliotecas. Servicios de Información Electrónica: Biblioteca Digital. [Tríptico informativo]. México: DGB, 2003.

9. Universidad Nacional Autónoma de México. Dirección General de Bibliotecas. Comité de libro electrónico. [Folleto]. México: DGB, 2003.

10. Diccionario de la lengua española. $2^{\mathrm{a}} \mathrm{ed}$. México: Ediciones Culturales Internacionales, 1991.

\section{Obras consultadas}

Diccionario de la lengua española. $2^{\mathrm{a}}$ ed. México: Ediciones Culturales Internacionales, 1991.

Hernández Mondragón, Alma Rosa, [et al.]. "Perspectivas en el manejo de información electrónica en la UNAM”. En: Reunión de revistas académicas y de investigación, 1997, pp. 82-118.

Lugo Hubp, Margarita; Orozco Aguirre, Aurelia. "La revista electrónica en la UNAM: retos presentes y futuros". En: Biblioteca universitaria: revista de la Dirección General de Bibliotecas de la UNAM, 2003, julio-diciembre, v. 6, n. 2, pp. 138-151.
Orozco Aguirre, Aurelia; Lugo Hubp, Margarita. Políticas de suscripción y renovación de bases de datos especializadas/UNAM, Subdirección de Servicios Especializados y Departamento de Suscripciones. México, 2003. [Documento interno de trabajo].

Rodríguez Gallardo, Adolfo. "La experiencia de la UNAM en la selección, adquisición y administración de revistas electrónicas de texto completo". En: Infodiversidad, 2001, v. 3, pp. 21-35.

"El sistema bibliotecario de la UNAM en cifras. Radiografía del Sistema 2001". En: Biblioteca universitaria: revista de la Dirección General de Bibliotecas de la UNAM, 2002, enero-junio, v. 5, n. 1, pp. 61-65.

Universidad Nacional Autónoma de México. Agenda estadística 2003. México: UNAM, 2003.

Universidad Nacional Autónoma de México. Dirección General de Bibliotecas. Censo estadístico 2001. México, 2002.

Universidad Nacional Autónoma de México. Dirección General de Bibliotecas. Comité de libro electrónico [folleto]. México: DGB, 2003.

Universidad Nacional Autónoma de México. Dirección General de Bibliotecas. Departamento de Suscripciones. Concentrado de gastos. México, 1999. [Documento interno de trabajo].

Universidad Nacional Autónoma de México. Dirección General de Bibliotecas. Servicios de Información Electrónica: Biblioteca Digital [tríptico informativo]. México: DGB, 2003.

Margarita Lugo Hubp, Dirección General de Bibliotecas de la Universidad Nacional Autónoma de México. mlugo@servidor.unam.mx

Raúl Novelo Peña, Biblioteca del Instituto de Investigaciones en Matemáticas Aplicadas y en Sistemas de la Universidad Nacional Autónoma de México.

rnovelo@leibniz.iimas.unam.mx 